\title{
Design of LPF using Hi-Lo interdigital DGS slot
}

\author{
Yayun Zhanga), Long Jin, and Lei Li \\ Research Institute of Electronic Science and Technology University of Electronic \\ Science and Technology of China (UESTC), \\ No. 2006, Xiyuan AveWest Hi-Tech Zone, 611731 Chengdu, Sichuan, P. R. China \\ a) zhangyayun90@126.com
}

\begin{abstract}
A low pass filter (LPF) using High-Low impedance line (Hi-Lo) interdigital Defected Ground Structure (DGS) with compact size and large stopband is presented in this paper. Both of the two characteristics are benefit from the introduction of the high impedance line in the conventional interdigital DGS, which can decrease the transmission zeros of the DGS without changing its overall size. To better explain the principle of the proposed DGS, the equivalent circuit is also built and extracted.
\end{abstract}

Keywords: Hi-Lo interdigital DGS, compact, wide stopband

Classification: Microwave and millimeter wave devices, circuits, and systems

\section{References}

[1] D. Ahn, J.-S. Park, C.-S. Kim, J. Kim, Y. Qian and T. Itoh: IEEE Trans. Microw. Theory Techn. 49 (2001) 86. DOI:10.1109/22.899965

[2] Y. Yang, Y. He and L. Sun: 2014 IEEE International Symposium on RadioFrequency Integration Technology (RFIT) (2014) 1. DOI:10.1109/RFIT.2014. 6933260

[3] A. B. Abdel-Rahman, A. K. Verma, A. Boutejdar and A. S. Omar: IEEE Trans. Microw. Theory Techn. 52 (2004) 1008. DOI:10.1109/TMTT.2004.823587

[4] A. Boutejdar, A. Elsherbini and A. Omar: Microwave Conference, European (2007) 842. DOI:10.1109/EUMC.2007.4405324

[5] Y. Han, B. Yang and Q. Guo: 2013 IEEE International Symposium on Electromagnetic Compatibility (EMC) (2013) 636. DOI:10.1109/ISEMC.2013. 6670489

[6] M. K. Mandal and S. Sanyal: IEEE Microw. Wireless Compon. Lett. 16 (2006) 93. DOI:10.1109/LMWC.2005.863192

[7] C.-S. Kim, J.-S. Park, D. Ahn and J.-B. Lim: IEEE Microw. Guided Wave Lett. 10 (2000) 131. DOI:10.1109/75.846922

[8] Q. Chen and J. Xu: Electron. Lett. 46 (2010) 1447. DOI:10.1049/el.2010.2251

[9] S. Liu, J. Xu and Z. Xu: Electron. Lett. 51 (2015) 1343. DOI:10.1049/el.2015. 0721

[10] M. Kufa and Z. Raida: IEEE Microw. Wireless Compon. Lett. 17 (2007) 586. DOI:10.1109/LMWC.2007.901769

[11] J.-L. Li, S.-W. Qu and Q. Xue: Electron. Lett. 45 (2009) 110. DOI:10.1049/ el:20093246 


\section{Introduction}

DGS is first introduced by Dal Ahn [1]. By etching a defected pattern in the ground, the shield current distribution in the ground plane is disturbed and excellent bandgap characteristic in some frequency bands is obtained with only one or more unit DGS cells. This useful characteristic attracts researcher's attention. Varieties of DGS structures, such as symmetrical, asymmetrical and periodic structures, have been designed and used in microwave and millimeter wave circuit, especially in filter applications $[2,3,4,5,6,7,8]$. Filters based on uniform interdigital DGS slot, with sharp roll-off and wide stopband are also reported $[9,10]$.

In the uniform interdigital DGS presented in $[9,10]$, transmission zeros are adjusted mainly by tuning the length of the finger. To get lower transmission zeros, the finger length increases, which enlarges the size of LPF. While reducing the finger width can increase the inductance, but it will also decrease the couple capacitance, resulting in the resonant frequency unchanged or even increasing. That's means the width of finger should be large enough to keep the couple capacitance at certain level. Then the Hi-Lo interdigital DGS is proposed in the paper. The low impedance line of the finger keeps the couple capacitance at certain level, while the high impedance one increases the inductance, finally resulting in the lower working frequency without enlarging the DGS size. A LPF using two cells of the proposed DGS has been designed, fabricated and measured. The measured results show good roll-off performance and wide stopband with large size reduction.

\section{Characters of the DGS cell}

The geometric structure of the proposed DGS and its equivalent RLC circuit are shown in Fig. 1. As shown in Fig. 1a, the proposed DGS is consist of three open Hi-Lo impedance fingers with same size in the ground plane. Each finger is consists of a low impedance line (line1) and a high impedance line (line2). The finger becomes uniform finger when the width of line1 and line2 is equal. The DGS is fed by a low impedance line in the center of the top side, while the $50 \mathrm{Ohm}$ characteristic impedance line is used to lead out properly. Fig. $1 \mathrm{~b}$ gives the RLC equivalent circuit. To model the DGS cell as more accurate as possible, the differences of the electromagnetic field at the edge and the center of the DGS cell are considered here, which are reflected in the different values of the R, L, C components. It should be noted that the radiation loss of the DGS is modeled by the resistance. The whole size $\mathrm{a}, \mathrm{b}$ control the inductance $\mathrm{L} 1$ and resistance $\mathrm{R} 1$, couple capacitance $\mathrm{C} 1, \mathrm{C} 2$, $\mathrm{C} 5$ and $\mathrm{C} 7$ are decided by g1, g2, g3 respectively, while $\mathrm{C} 3$ and $\mathrm{C} 8$ mainly depends on W2. Resonate pairs of RLC1 and RLC3 are decided by line1, while Line2 controls resonate pairs of RLC2 and RLC4. 


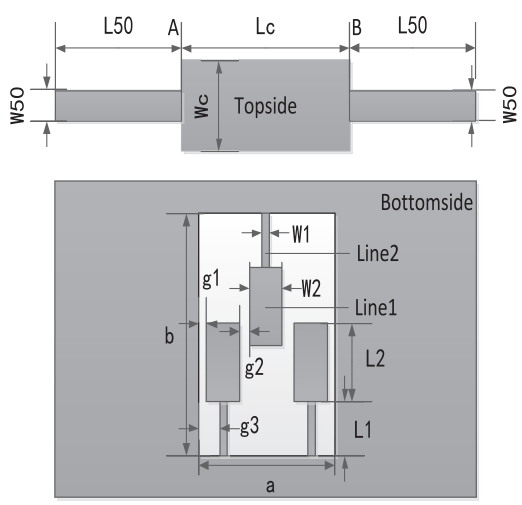

(a) Proposed Hi-Lo interdigital DGS

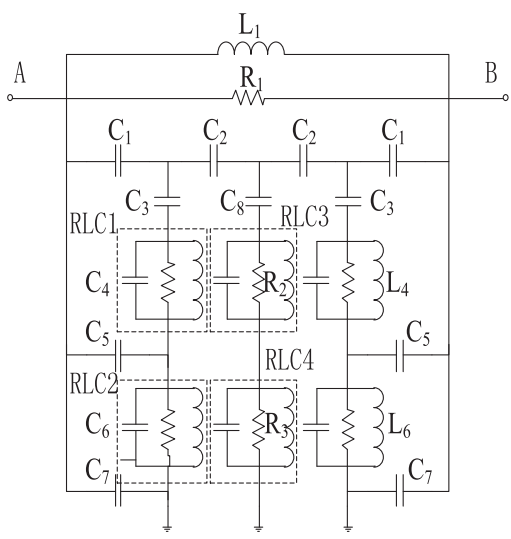

(b) The R-L-C equivalent circuit

Fig. 1. Schematic and R-L-C equivalent circuit of the proposed DGS.

The dimensions of the DGS cell in Fig. 1a are shown in Table I. To prove the effect of the W1, simulation with varied $\mathrm{W} 1$ from $0.2 \mathrm{~mm}$ to $1 \mathrm{~mm}$ is performed. Results are given in Fig. 2. It should be noted that Hi-Lo interdigital DGS becomes uniform one when $\mathrm{W} 1=1 \mathrm{~mm}$, which acts as a reference to prove the effect of the $\mathrm{W} 1$ on the resonant frequency. It can be seen that lower two frequencies move to the low side when W1 decreases, while keeping the highest one unchanged (less obvious), which is helpful in enlarging the stopband.

Table I. Parameters of the proposed Hi-Lo interdigital DGS cell in millimeters (see Fig. 1a).

\begin{tabular}{|c|c|c|c|c|c|c|c|c|c|c|c|}
\hline W50 & L50 & Wc & Lc & a & b & W1 & L1 & W2 & L2 & g1 & g2 \\
\hline 1.1 & 3.0 & 3.0 & 6.2 & 4.2 & 12.8 & 0.4 & 4.1 & 1.0 & 3.0 & 0.2 & 0.4 \\
\hline
\end{tabular}

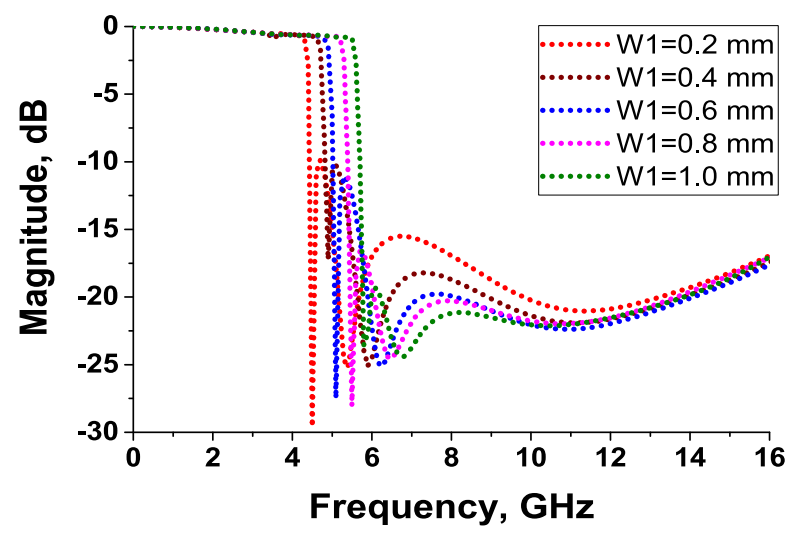

Fig. 2. Simulated S21 (dB) of proposed DGS with varied line width W1. 
Table II. The values of R, L, C components under the case of Table I (see Fig. 1b).

\begin{tabular}{|c|c|c|c|c|c|c|}
\hline & L1 & C1 & C2 & C3 & C5 & C7 \\
\hline L $(\mathrm{nH})$ & 2.010 & - & - & - & - & - \\
\hline $\mathrm{R}(\mathrm{Ohm})$ & - & - & - & - & - & - \\
\hline $\mathrm{C}(\mathrm{pF})$ & - & 0.820 & 0.600 & 0.186 & 0.180 & 0.115 \\
\hline & $\mathrm{C} 8$ & $\mathrm{R} 1$ & LRC1 & LRC2 & LRC3 & LRC4 \\
\hline $\mathrm{L}(\mathrm{nH})$ & - & - & $10.100 \mathrm{e}-3$ & 0.958 & $10.100 \mathrm{e}-3$ & 1.397 \\
\hline $\mathrm{R}(\mathrm{Ohm})$ & - & 560.720 & 18.970 & 3920.000 & 18.970 & 522.009 \\
\hline $\mathrm{C}(\mathrm{pF})$ & 2.600 & - & 210.090 & 0.749 & 210.090 & 0.190 \\
\hline
\end{tabular}

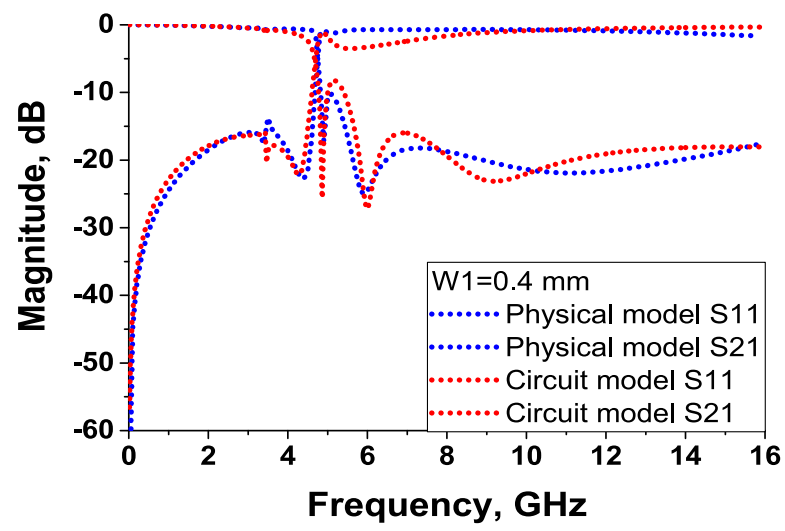

Fig. 3. Simulated S parameters of physical model and circuit model.

\section{Design and measurements of the LPF}

With the help of electromagnetic simulator HFSS 15.0, a LPF has been fabricated using RO4003C with $0.508 \mathrm{~mm}$ thick and relative dielectric constant $\varepsilon_{r}$ of 3.55. The design step of LPF can be divided into four steps:

I: Getting the original size of the simplest DGS, which has no fingers.

Parameters $\mathrm{a}$ and $\mathrm{b}$ are used to set the highest zero and cut off frequency, which need not very accurate. In order to make two or more cells cascade easier, $b$ should be much bigger than a. Thus, big width of the feed line (Wc) is always needed to feed DGS cell effectively. Furthermore, the zero obtained in this step is less perfect due to the large length of the DGS cell (b)

II: The introduction of uniform fingers.

Keeping the whole size of DGS unchanged, other two attenuation poles are introduced by the fingers. It should be noted that the finger width needs large enough to keep the open-end couple capacitance at certain level, which means the small values of $\mathrm{g} 1$ and $\mathrm{g} 2$.

III: Decreasing the width of line2.

High impedance line is introduced here to further decrease the lower transmission zeros introduced in step II.

IV: Cascade of the DGS cells and optimization

The cascade of DGSs designed upstairs can be simply finished by a transmission line, the size of which is obtained mainly by simulation. Then optimization is performed to get the best performance. 
The geometric configuration of the proposed LPF is shown in Fig. 4, which includes the Hi-Lo impedance transmission line on the topside and two proposed DGS units in the ground plane. The dimensions of the LPF are shown in Table III.
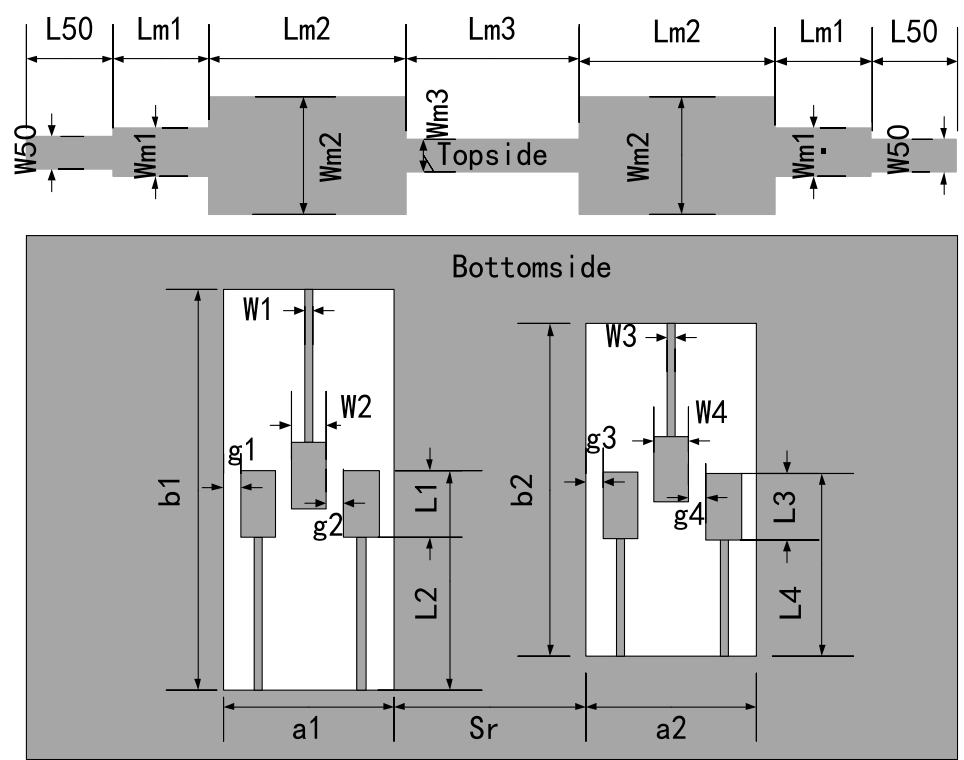

(a) Schematic diagram

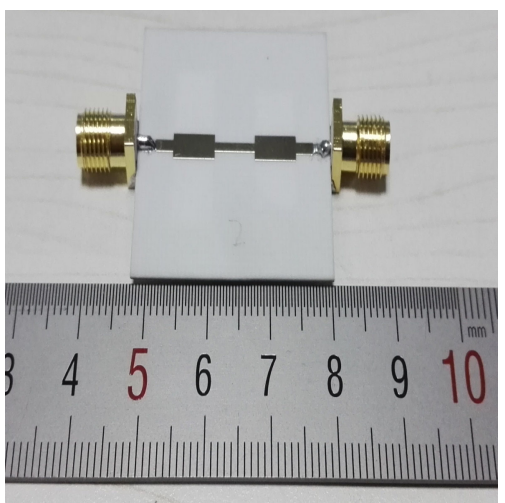

(b) Top of the proposed filter

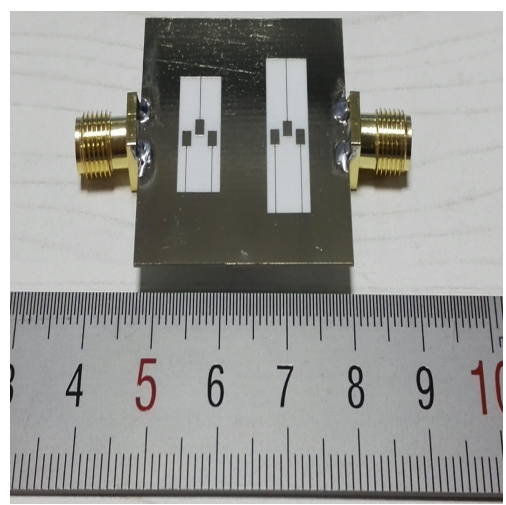

(c) Bottom of the proposed filter

Fig. 4. Schematic diagram and photographs of the proposed filter.

Table III. Dimensions of the proposed LPF in millimeters (see Fig. 4).

\begin{tabular}{|c|c|c|c|c|c|c|c|c|c|c|c|c|}
\hline W50 & L50 & Wm1 & Lm1 & Wm2 & Lm2 & Wm3 & Lm3 & a1 & a2 & b1 & b2 & g1 \\
\hline 1.1 & 3.0 & 1.4 & 2.9 & 3.0 & 6.6 & 1.1 & 6.8 & 6.6 & 6.6 & 18.8 & 14 & 0.6 \\
\hline $\mathrm{g} 2$ & $\mathrm{~g} 3$ & $\mathrm{~g} 4$ & $\mathrm{~L} 1$ & $\mathrm{~L} 2$ & $\mathrm{~L} 3$ & $\mathrm{~L} 4$ & $\mathrm{~W} 1$ & $\mathrm{~W} 2$ & $\mathrm{~W} 3$ & $\mathrm{~W} 4$ & $\mathrm{Sr}$ & - \\
\hline 0.6 & 0.6 & 0.6 & 2.0 & 9.9 & 2.0 & 7.3 & 0.1 & 1.4 & 0.1 & 1.4 & 7.2 & - \\
\hline
\end{tabular}

Simulation and measurement were accomplished using EM simulation HFSS15.0 and Agilent E8363B network analyser respectively. The simulation and measurement results are shown in Fig. 5. Changes are found between simulated and the measured results, which may be caused by the fabrication error and the difference in dielectric constant between simulated and real one. As can be seen, 
the measured $3 \mathrm{~dB}$ cutoff frequency $\left(f_{c}\right)$ is $2.87 \mathrm{GHz}$, the in-band return loss is better than $-14 \mathrm{~dB}$. The transition band is from $2.87 \mathrm{GHz}$ to $3.05 \mathrm{GHz}$ with $-3.05 \mathrm{~dB}$ to $-20.25 \mathrm{~dB}$, showing a $95.55 \mathrm{~dB} / \mathrm{GHz}$ roll-off. Stopband with rejection better than $20 \mathrm{~dB}$ is from $3.05 \mathrm{GHz}$ to more than $16 \mathrm{GHz}$, which is bigger than $4.51 f_{c}$. The effective size of LPF is $26.67 * 30.00 \mathrm{~mm}^{2}\left(0.4897 * 0.5407 \lambda \mathrm{g}^{2}\right)$ if the length of $50 \mathrm{Ohm}$ characteristic transmission line $(6 \mathrm{~mm})$ is excluded. Comparing with LPF based on the uniform interdigital DGS in [9], about $77.7 \%$ size reduction as well as more than $4.51 f_{c}$ stopband with rejection better than $-20 \mathrm{~dB}$ is achieved. Comparison with some reported LPF is given in Table IV.

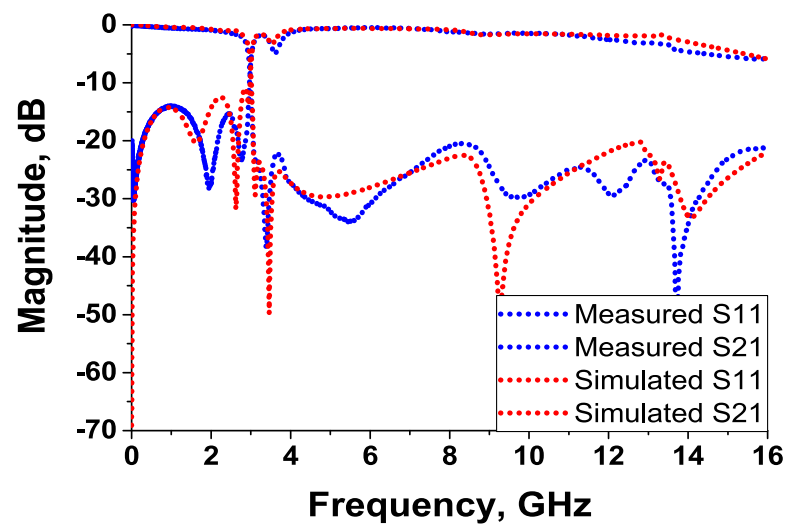

Fig. 5. Simulated S parameters of physical model and circuit model.

Table IV. Performance comparisons published filters and proposed one.

\begin{tabular}{|c|c|c|c|c|}
\hline Ref. & $\begin{array}{c}\text { Roll-off } \\
\text { rate [11] }\end{array}$ & $\begin{array}{c}\text { Relative stopband } \\
\text { bandwidth [11] }\end{array}$ & $\begin{array}{c}\text { Suppression } \\
\text { factor [11] }\end{array}$ & $\begin{array}{c}\text { Normalized } \\
\text { circuit size }\end{array}$ \\
\hline$[8]$ & 130 & 1.382 & 2.5 & $1.1866 * 0.6724$ \\
\hline$[9]$ & 171.5 & 1.124 & 2.5 & $1.177 * 0.9896$ \\
\hline$[10]$ & 15.5 & 0.896 & 2 & NG \\
\hline$[11]$ & 92.5 & 1.355 & 3 & NG \\
\hline This work & 95.5 & 1.359 & 4.51 & $0.5407 * 0.4807$ \\
\hline
\end{tabular}

\section{Conclusion}

A LPF based on Hi-Lo interdigital DGS has been designed, fabricated and measured. The measured results show good agreement with that of the simulation. Compared with the uniform one in [9], about $77.7 \%$ size reduction as well as more than $4.51 f_{c}$ stopband with rejection better than $-20 \mathrm{~dB}$ is achieved, which shows the potential of the proposed DGS in size reduction and applications working at low frequency. 